\title{
Research on Allocation of Charging Resources Based on Neural Network
}

\author{
Yiran Wu, ZiShang Wu, Dengyuan Yang, Xizhe Wang \\ Nanjing Foreign Language School Xianlin Campus, China
}

Keywords: BP Neural Network, Prediction

\begin{abstract}
For charging, it can be divided into indoor charging and outdoor charging. In this paper, through the establishment of neural network prediction model, power charging model, data analysis, entropy division and other models, the recent use of public resources data is obtained. Finally, according to the analysis results of these models, some suggestions on charging are put forward in the school newspaper. Electric energy consumption in public places mainly includes outdoor charging cars and indoor charging mobile phones. Based on the data of automobile sales and mobile phone sales in recent years, according to the rising trend of the number of mobile phones and electric vehicles, we can draw the conclusion that the power consumption is on the rise. Then use the neural network prediction model to predict the development trend in the next decade: with the increase of car and mobile phone sales, the demand for public facilities charges increases.
\end{abstract}

\section{Restatement of problems}

\subsection{Background of problem}

In our social life, we live in a mobile electronic world. Every day we "plug in" to charge our electronic devices. These electronic products may be from small items (mobile phones) to large items (electric vehicles) in our own home. Our family is likely to be responsible for purchasing charging devices and then paying the power we use to the power supplier.

\subsection{Question raise}

1 Discuss how such energy consumption has changed in recent years and how it will continue to change to determine the impact and requirements of these growing energy (electricity) and charging demands on public places.

2 Using the impacts and needs identified, build a model for the costs of increased demand for public places and energy use to discuss the scope of these costs and how to pay them.

3 Discuss the changes of the model in different types of public places (such as schools and cafes and airports and shopping centers)?

4 What measures should be explored to reduce the increased cost of energy use in public places how will the implementation of these plans adjust the cost model?

5 Write a one-page article for the school newspaper describing the findings and suggestions.

\section{Problem analysis}

The research will be divided into two parts: the outdoor charging car and the indoor charging mobile phone. The car sales data and the mobile phone sales data in recent years are queried, and the data image shows that the power consumption is on the rise. Neural network prediction is used to predict the changes in the next ten years. As car and mobile phone sales increase, the demand for charging public facilities increases. Due to the increased demand for charging, charging facilities are required. According to indoor and outdoor demand ratio, indoor and outdoor density, indoor and outdoor space. Establish the model of electric charge system, get the range of charge and the way of payment. In different places, according to its actual use function, the weight of indoor and outdoor charging is determined by using the entropy decentralization model. According to the conclusion of model 2, the change of schools, cafes, airports and shopping centers is analyzed. 


\section{Model hypotheses}

Hypothesis 1 assumes all the data are correct.

\section{Symbolic explanation}

\begin{tabular}{cc}
\hline Symbo & Explain \\
$\mathrm{l}$ & \\
\hline$x_{i}^{\prime}$ & Input variables of multiple linear regression \\
$y_{i}^{\prime}$ & Output variables of multiple linear regression \\
$x_{i}$ & Input variables of multivariate nonlinear regression \\
$y_{i}$ & Output variables of multiple linear regression \\
$\beta$ & Regression coefficient \\
$\hat{\beta}$ & Regression coefficient estimate \\
$\hat{y}$ & Output variable estimate \\
$\mathrm{Q}$ & Sum of squares of residuals \\
$\mathrm{E}$ & Fitting error \\
$X^{(0)}$ & Primordial sequence \\
$A$ & Judgement matrix \\
\hline
\end{tabular}

\section{Method}

\subsection{Model building}

The basic principle of BP (back propagation) neural network is to transform an input vector (provided by training samples) through a series of transformations in the hidden layer, and then get an output vector, so as to realize a mapping relationship between input data and output data. The forward propagation of input information and the backward propagation of output error constitute the information cycle of BP network. BP algorithm modifies the connection weight coefficient of each neuron connection according to the output error, and its purpose is to make the output error reach the pre remembered range. BP network is a tutor learning network because it needs the error between the actual output and the expected output to determine whether to modify the connection weight coefficient between neurons. Among them, the expected output is the "mentor" in the sense of the network. BP network has a symmetrical network structure. In the small BP network, each processing unit of the output has the same excitation function.

\subsubsection{Topological structure of BP neural network}

BP neural network model is a three-layer network. Its topology can be divided into input layer, output layer and hide layer. Among them, the input layer and the output layer have more critical significance, so it can also be a two-layer network structure (put the hidden layer into the input layer, or remove the hidden layer). Each layer consists of many simple neurons that can perform parallel operations. These neurons are very similar to those in biological systems, but their parallelism is not as high as that of biological neurons. BP neural network is a feedforward network, so it has the characteristics of a feedforward network: all the neurons in the adjacent two layers are connected, but the neurons in the same layer cannot be connected. Although the structure of a single neuron is very simple and its function is minimal, the network system composed of a large number of neurons can achieve wealthy and colorful functions and solve many complex problems. Neural network can process information in parallel and store information in distributed way, so it can be seen that it is a kind of nonlinear dynamic system, which is described above. 
The network topology of BP network is a multi-layer feed-forward network structure in the computer economy network. In the topological structure of BP network, the number of layers of network and the number of input and output nodes are determined by the problem waiting to be solved. The most important thing is to determine the number of layers of the hidden layer and the number of nodes in the hidden layer.

The hidden layer is the middle layer between the input layer and the output layer of the neural network, which can be one or more layers

It is also regarded as an internal representation of input patterns in neural networks. Its function is to extract the features that are different from other input modes and transfer the extracted features to the output layer. Finally, the output layer makes a judgment on them, which is mainly the process of judging whether this type of input mode works with other input modes and the same hidden layer, that is, the process of extracting input mode features. In fact, it realizes the adjustment process of the connection weight coefficient between the input layer and the hidden layer, which is a "self-organizing process". Therefore, in the process of network learning and training, the connection weight coefficient between layers plays a role of bridge and carries out "feature transfer".

\subsubsection{The basic principle of BP neural network}

BP neuron (node), similar to artificial neuron, mainly imitates the three most important characteristics of the biological neurons: weighting, summation and transfer.

Among them $x_{1}, x_{2} \ldots x_{i} \cdots x_{n}$, the vector represents the input from the $1,2, \cdots i \cdots n$ neuron respectively; this vector represents the connection weight coefficient between the neuron in the previous layer of the network and the j-th neuron in this layer; bi is the threshold value; $f()$ is the transfer function ; it is the output of the $\mathrm{j}$-th neuron

$$
S_{j}=\sum_{i=1}^{n} w_{j i} \cdot x_{i}+b_{j}=W_{j} X+b_{j}
$$

Among them, $X=\left[x_{1} x_{2} \cdots x_{i} \cdots x_{n}\right]^{T}, W=\left[w_{1} w_{2} \cdots w_{i} \cdots w_{n}\right]$.

If you let $x_{0}=1, w_{j 0}=b_{j}, \mathrm{X}$ and $W_{j}$ Include $x_{0}$ and $w_{j 0}$,be:

$$
\begin{gathered}
X=\left[x_{0} x_{1} x_{2} \cdots x_{i} \cdots x_{n}\right]^{T} \\
W=\left[w_{0} w_{1} w_{2} \cdots w_{i} \cdots w_{n}\right] \\
d_{k}=\left[d_{k 1}, d_{k 2}, \cdots d_{k p}\right],(k=1,2, \cdots n) \\
X_{k}(k=1,2, \cdots, n)
\end{gathered}
$$

Then, the net input $S_{j}$ of node j can be expressed by the following formula:

$$
S_{j}=\sum_{i=1}^{n} w_{j i} \cdot x_{i}=W_{j} X
$$

After the net input $S_{j}$ passes through the transfer function $f(\bullet)$, the output $y_{j}$ of the j-th neuron can be obtained:

$$
y_{i}=f\left(S_{j}\right)=f\left(\sum_{i=0}^{n} w_{j i} \cdot x_{i}\right)=f\left(W_{j} X\right)
$$

In the above formula, $f(\bullet)$ is a monotonically increasing bounded function, which has an upper limit, i.e. the maximum value, because the signals transmitted by neurons cannot be infinite.

\subsubsection{Principle of BP neural network algorithm}

The basic BP algorithm includes two aspects: forward propagation of signal and BP of error. That is to say, the actual output is calculated according to the direction from input to output, while the correction of weight and threshold is carried out from the direction from output to input. 


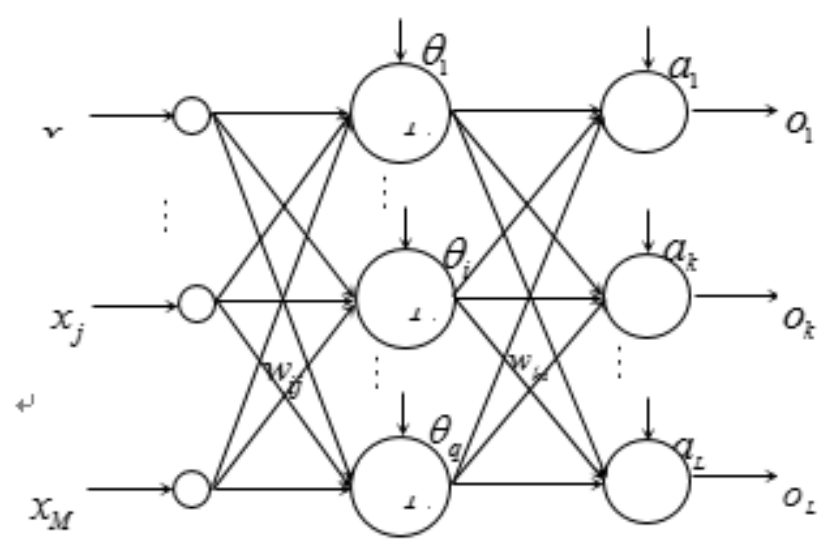

Figure 1 BP network structure

(1) Forward propagation process of signal Input Net of the i th node of the hidden layer:

$$
n e t_{i}=\sum_{j=1}^{M} w_{i j} x_{j}+\theta_{i}
$$

Output yi of the i th node of the hidden layer:

$$
y_{i}=\phi\left(\text { net }_{i}\right)=\phi\left(\sum_{j=1}^{M} w_{i j} x_{j}+\theta_{i}\right)
$$

Input node of node $\mathrm{k}$ in output layer:

$$
n e t_{k}=\sum_{i=1}^{q} w_{k i} y_{i}+a_{k}=\sum_{i=1}^{q} w_{k i} \phi\left(\sum_{j=1}^{M} w_{i j} x_{j}+\theta_{i}\right)+a_{k}
$$

Output ok of the k-th node of the output layer:

$$
o_{k}=\psi\left(n e t_{k}\right)=\psi\left(\sum_{i=1}^{q} w_{k i} y_{i}+a_{k}\right)=\psi\left(\sum_{i=1}^{q} w_{k i} \phi\left(\sum_{j=1}^{M} w_{i j} x_{j}+\theta_{i}\right)+a_{k}\right)
$$

(2) BP process of error

The BP of the error, that is, the output error of each layer of neurons is calculated from the output layer first, and then the weight and threshold of each layer are adjusted according to the error gradient descent method, so that the final output of the modified network can meet the near-term expected value.

For each sample P, the quadratic error criterion function is EP:

$$
E_{p}=\frac{1}{2} \sum_{k=1}^{L}\left(T_{k}-o_{k}\right)^{2}
$$

The total error criterion function of the system for $\mathrm{P}$ training samples is:

$$
E=\frac{1}{2} \sum_{p=1}^{P} \sum_{k=1}^{L}\left(T_{k}^{p}-o_{k}^{p}\right)^{2}
$$

According to the error gradient descent method, the correction value of output layer weight $\Delta$ wki, output layer threshold value $\Delta \mathrm{ak}$, hidden layer weight value $\Delta$ wij, hidden layer threshold value is modified in turn $\Delta \boldsymbol{\theta}_{\boldsymbol{i}}$ 。

$$
\Delta w_{k i}=-\eta \frac{\partial E}{\partial w_{k i}} \quad \Delta a_{k}=-\eta \frac{\partial E}{\partial a_{k}} \quad \Delta w_{i j}=-\eta \frac{\partial E}{\partial w_{i j}} \quad \Delta \theta_{i}=-\eta \frac{\partial E}{\partial \theta_{i}}
$$

Output layer weight adjustment formula:

$$
\Delta w_{k i}=-\eta \frac{\partial E}{\partial w_{k i}}=-\eta \frac{\partial E}{\partial n e t_{k}} \frac{\partial n e t_{k}}{\partial w_{k i}}=-\eta \frac{\partial E}{\partial o_{k}} \frac{\partial o_{k}}{\partial n e t_{k}} \frac{\partial n e t_{k}}{\partial w_{k i}}
$$

Output layer threshold adjustment formula: 


$$
\Delta a_{k}=-\eta \frac{\partial E}{\partial a_{k}}=-\eta \frac{\partial E}{\partial n e t_{k}} \frac{\partial n e t_{k}}{\partial a_{k}}=-\eta \frac{\partial E}{\partial o_{k}} \frac{\partial o_{k}}{\partial n e t_{k}} \frac{\partial n e t_{k}}{\partial a_{k}}
$$

Hidden layer weight adjustment formula:

$$
\Delta w_{i j}=-\eta \frac{\partial E}{\partial w_{i j}}=-\eta \frac{\partial E}{\partial n e t_{i}} \frac{\partial n e t_{i}}{\partial w_{i j}}=-\eta \frac{\partial E}{\partial y_{i}} \frac{\partial y_{i}}{\partial n e t_{i}} \frac{\partial n e t_{i}}{\partial w_{i j}}
$$

Hidden layer threshold adjustment formula:

$$
\Delta \theta_{i}=-\eta \frac{\partial E}{\partial \theta_{i}}=-\eta \frac{\partial E}{\partial n e t_{i}} \frac{\partial n e t_{i}}{\partial \theta_{i}}=-\eta \frac{\partial E}{\partial y_{i}} \frac{\partial y_{i}}{\partial n e t_{i}} \frac{\partial n e t_{i}}{\partial \theta_{i}}
$$

And because:

$$
\begin{gathered}
\frac{\partial E}{\partial o_{k}}=-\sum_{p=1}^{P} \sum_{k=1}^{L}\left(T_{k}^{p}-o_{k}^{p}\right) \\
\frac{\partial n e t_{k}}{\partial w_{k i}}=y_{i} \frac{\partial n e t_{k}}{\partial a_{k}}=1 \frac{\partial n e t_{i}}{\partial w_{i j}}=x_{j} \frac{\partial n e t_{i}}{\partial \theta_{i}}=1 \\
\frac{\partial E}{\partial y_{i}}=-\sum_{p=1}^{P} \sum_{k=1}^{L}\left(T_{k}^{p}-o_{k}^{p}\right) \cdot \psi^{\prime}\left(\text { net }_{k}\right) \cdot w_{k i} \\
\frac{\partial y_{i}}{\partial n e t_{i}}=\phi^{\prime}\left(n e t_{i}\right) \\
\frac{\partial o_{k}}{\partial n e t_{k}}=\psi^{\prime}\left(\text { net }_{k}\right)
\end{gathered}
$$

So we get the following formula at last:

$$
\begin{gathered}
\Delta w_{k i}=\eta \sum_{p=1}^{P} \sum_{k=1}^{L}\left(T_{k}{ }^{p}-o_{k}{ }^{p}\right) \cdot \psi^{\prime}\left(\text { net }_{k}\right) \cdot y_{i} \\
\Delta a_{k}=\eta \sum_{p=1}^{P} \sum_{k=1}^{L}\left(T_{k}{ }^{p}-o_{k}{ }^{p}\right) \cdot \psi^{\prime}\left(\text { net }_{k}\right) \\
\Delta w_{i j}=\eta \sum_{p=1}^{P} \sum_{k=1}^{L}\left(T_{k}^{p}-o_{k}^{p}\right) \cdot \psi^{\prime}\left(\text { net }_{k}\right) \cdot w_{k i} \cdot \phi^{\prime}\left(\text { net }_{i}\right) \cdot x_{j} \\
\Delta \theta_{i}=\eta \sum_{p=1}^{P} \sum_{k=1}^{L}\left(T_{k}^{p}-o_{k}^{p}\right) \cdot \psi^{\prime}\left(\text { net }_{k}\right) \cdot w_{k i} \cdot \phi^{\prime}\left(\text { net }_{i}\right)
\end{gathered}
$$

The specific steps of BP algorithm are as follows: 


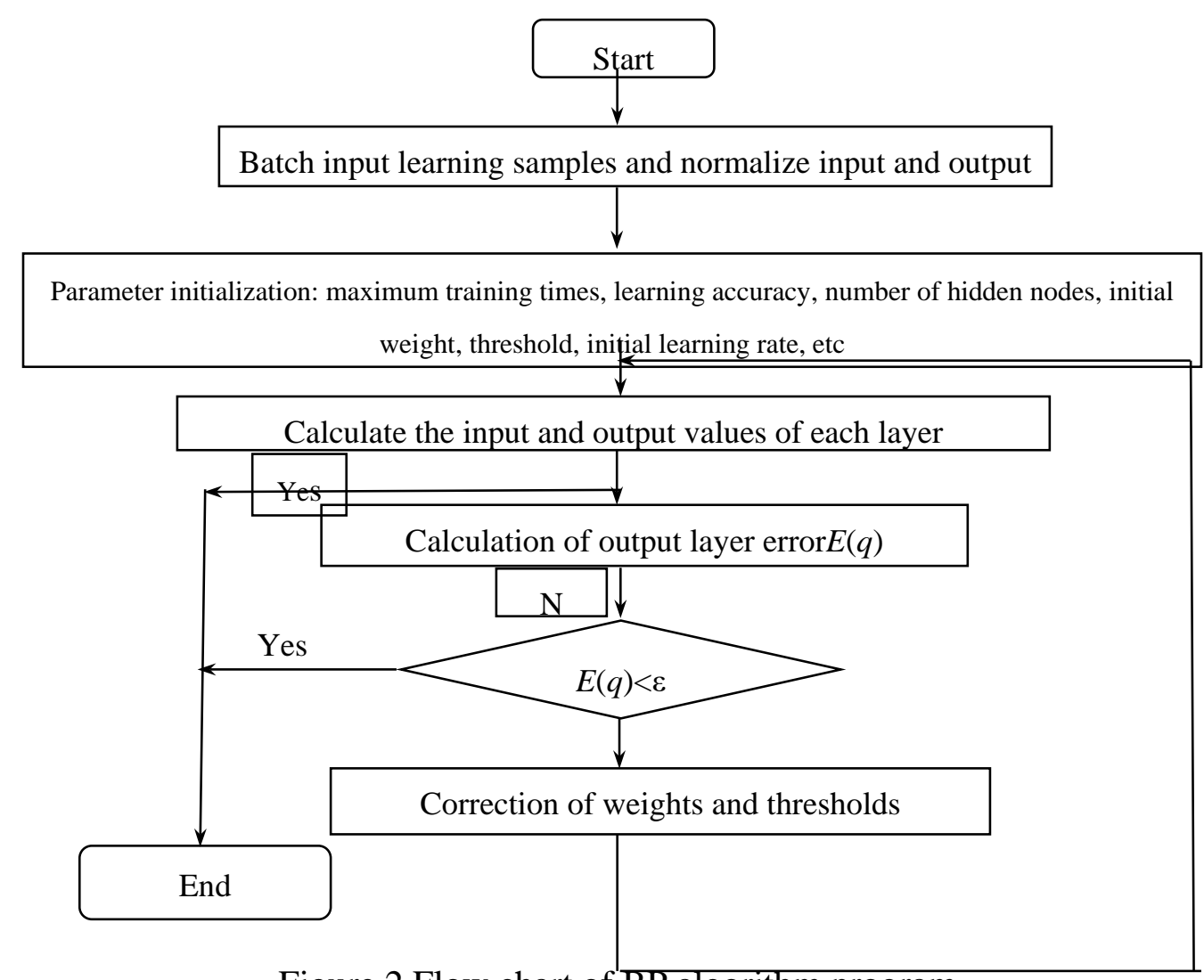

Figure 2 Flow chart of BP algorithm program

\subsection{Model solution}

The least-square method is used to fit the data and get the linear fitting equation. According to the linear equation, get the prediction data, and analyze the error of the prediction data, get the error proportion.

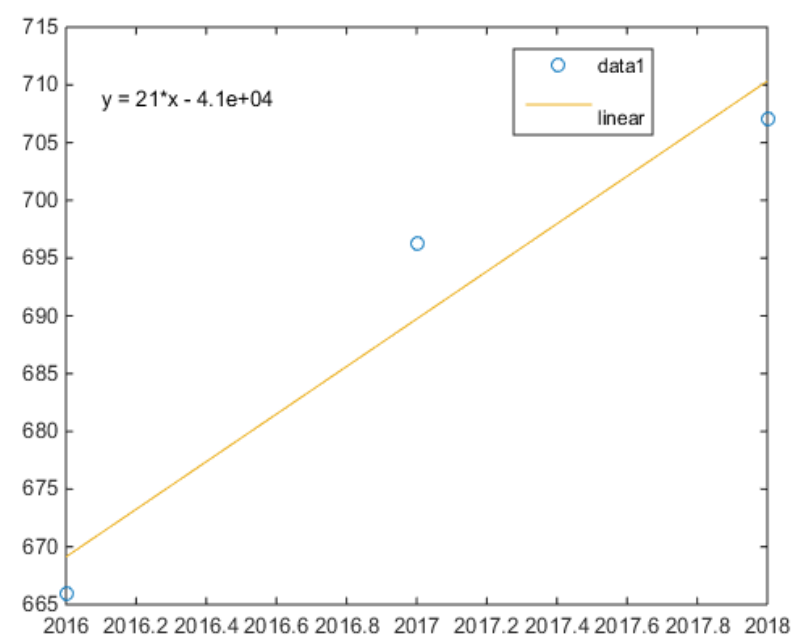

Figure 3 Mobile phone sales data forecast

The predicted data and error analysis are shown in Table 2 below. 
Table 1 Error analysis of mobile phone sales forecast

\begin{tabular}{|c|c|c|c|}
\hline Time & Total (million) & Forecast value (million) & Error(\%) \\
\hline 2016 & 665.9 & 669.1 & 0.05 \\
\hline 2017 & 696.2 & 689.5 & 1 \\
\hline 2018 & 707.1 & 710.1 & 0.5 \\
\hline 2019 & & 730.57 & \\
\hline 2020 & & 751.07 & \\
\hline 2021 & & 771.57 & \\
\hline
\end{tabular}

Put the electric vehicle data (see Appendix 1) queried by MIIT into the model, and use Matlab to establish the neural network model for solution. See Appendix 3 for the code, and get the prediction figure as shown in Figure 3

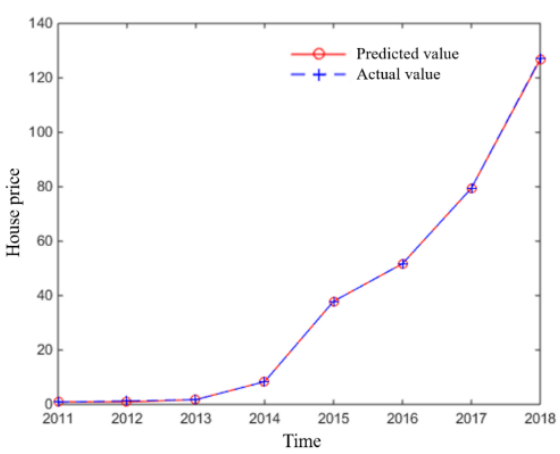

Figure 4 Prediction diagram of neural network

The predicted data and error analysis are shown in Table 2 below

Table 2 Sales volume and error analysis of new energy vehicles

\begin{tabular}{|c|c|c|c|}
\hline Time & New energy vehicles & Forecast value & Error(\%) \\
\hline 2011 & 8400 & 8400 & 0 \\
\hline 2012 & 12800 & 12800 & 0 \\
\hline 2013 & 17500 & 17500 & 0 \\
\hline 2014 & 83900 & 83900 & 0 \\
\hline 2015 & 379000 & 379000 & 0 \\
\hline 2016 & 517000 & 517000 & 0 \\
\hline 2017 & 794000 & 794000 & \\
\hline 2018 & 1270500 & 1270500 & \\
\hline 2019 & & 1530000 & \\
\hline 2020 & & 217000 & \\
\hline 2021 & & 275000 & \\
\hline 2022 & & 361000 & \\
\hline 2023 & & 436000 & \\
\hline 2024 & & 512000 & \\
\hline 2025 & & 605000 & \\
\hline 2026 & & 656000 & \\
\hline
\end{tabular}

\subsection{Summary}

It can be seen from the forecast data in Figure 1 and table 1 that the growth rate of mobile phones is relatively slow because they have passed the period of rapid growth. In the next three years, most people will take mobile phone with them when they use it. However, due to the lack of battery life, 
more and more smartphone functions, the power consumption is still not optimistic. Charging needs to be strengthened during out hours.

From 2013 to 2015, thanks to the policy support, the sales volume of new energy vehicles has increased several times. After the crazy growth period, the annual growth rate of new energy vehicles will continue to increase by about 50\% from 2016 to 2018. According to the predicted data, new energy vehicles will still maintain a high growth rate in the future this year. In the future, new energy vehicles will gradually replace the current gasoline vehicles, and the demand for charging piles will increase again in the next few years.

These are a big challenge for the number of charging equipment in the market. In many places, it will be inconvenient to charge, which will lead to the loss of customers and increase the loss of enterprises. Therefore, the public construction area needs to add more charging piles to reach the balance of the market.

\section{Acknowledgement}

These authors are contributed equally to this work.

\section{References}

[1] Zhang Lifeng. Prediction model and development strategy of China's energy supply and demand [D]. Capital University of economics and trade, 2006.

[2] Wang Shaohua. Research on supply and demand situation and development trend of power industry in China [D]. Harbin Engineering University, 2010.

[3] Wang Ronggui. Prediction of energy supply and demand in Guangdong Province and Research on development strategies [D]. Jinan University, 2011.

[4] Li Xiaobing. Research on prediction model of China's energy supply and demand based on system dynamics [D]. Inner Mongolia University of science and technology, 2015.

[5] Deng Zhiru. Prediction of energy supply and demand in China [D]. Harbin Engineering University, 2011.

[6] Jing Gang. Research on DSM of Shaanxi Electric Power Company [D]. Xi'an University of technology, 2006.

[7] Ding Dong. Research on power marketing strategy and law of supply and demand [D]. China Agricultural University, 2005.

[8] Huang Xiaoli. Research on the application of DSM in Jiangsu Province [D]. Jiangsu University, 2005.

[9] Ma Ning. Sales volume of new energy vehicles in policy "empty window period" [J]. Product reliability report, 2016 (04): 8-9.

[10] Chen Desheng, Zhang Yangjian, Zhang Guoliang, Li Hongxia. Prediction of power supply and demand in China in 2020 [J]. Energy, 2015 (02): 97-101.

[11] Yang Minying. Thinking on the change of power supply and demand situation [J]. Research on quantitative economy, technology and economy, 2004 (02): 35-38.

[12] Tang Chu. Analysis and prediction of influencing factors of mobile phone sales [D]. Hunan University, 2017.

[13] Yu Shanjin. Power cost optimization of data center based on price in smart grid environment [D]. China University of science and technology, 2014. 